\title{
BOUNDED, CONSERVATIVE, LINEAR OPERATORS AND THE MAXIMAL GROUP
}

\author{
E. P. KELLY, JR. AND D. A. HOGAN
}

\begin{abstract}
Let $V$ denote a Banach space over the reals, $B[V]$ the bounded linear operators on $V, f$ a linear functional defined on a complete subspace, $(f)$, of $V$. A conservative operator is an element of the set $\mathscr{T}_{f}=\{T \mid T \in B[V], T((f)) \subseteq(f)\}$. In this setting this paper extends some of the results of a recent paper by Rhoades [Triangular summability methods and the boundary of the maximal group, Math. Z. 105 (1968), 284-290]. In this setting necessary and sufficient conditions are proven for $T \in \mathscr{T}_{f}$ to be in the maximal group of invertible elements, $\mathscr{M}$. Sufficient conditions are proven for $T \in \mathscr{T}_{f}$ to be in the boundary, $\mathscr{B}$, of $\mathscr{M}$. It is proven that $\mathscr{B}$ is a multiplicative semigroup and if $(f)$ is nontrivial, then $\mathscr{B}$ is nonconvex. Two questions raised in the paper by Rhoades were answered.
\end{abstract}

1. Introduction. It is conventional to call a bounded, linear operator on the set of convergent sequences a conservative operator. In this paper a conservative operator means a bounded, linear operator, $T$, on a Banach space containing a complete subspace which is invariant under $T$. Let $V$ denote a Banach space over the reals, $B[V]$ the bounded linear operators on $V, f$ a linear functional defined on a complete subspace, $(f)$, of $V$. Also the set of conservative operators are denoted as $\mathscr{T}_{f}=\{T \mid T \in B[V]$, $T(f) \subseteq(f)\}$.

A recent paper by Rhoades [2] presented an investigation of some of the properties of the maximal group of invertible elements of the Banach algebra of conservative, infinite, triangular matrices. This paper is an extension of some of these properties to the setting in the previous paragraph. Let $\mathscr{M}$ denote the maximal group of invertible elements of $\mathscr{T}_{f}$ and $\mathscr{B}$ the boundary of $\mathscr{M}$. Let the set of elements $T$ maps into $(f)$ be denoted by $(f)_{T}$, that is, $(f)_{T}=\{\xi \mid \xi \in V, T(\xi) \in(f)\}$.

Even though the results of this paper are motivated by [2], the structure of $\mathscr{T}_{f}$ in this paper is dependent on the linear functional $f$ and more basically the Banach space $V$. The role of $V$ is emphasized by considering the following cases:

(1) $V=\left\{\xi \mid \xi=\left(x_{1}, x_{2}, \cdots\right) \in c, x_{j}=0\right.$ for $\left.j>n\right\}$ where $c$ is the space of convergent real sequences, and $f$ the limit functional with domain $V$.

Received by the editors March 20, 1970 and, in revised form, October 25, 1970. AMS 1970 subject classifications. Primary 46L20, 47D10; Secondary 40C05. 
Then given $T \in B[c]$ such that $T$ is a triangle as in [2], $T \notin \mathscr{B}$ since $T$ is onto [3, p. 267];

(2) $V=c$, with the sup norm and $f$ as in (1). Then there are triangles $T \in B[c]$ such that $T \in \mathscr{B}$. Such a $T$ is the triangle $A=\left[a_{i j}\right]$, where $a_{i j}=1$ for $i=j$ and $i=j+2$ and $a_{i j}=0$ otherwise. Note that in this case $T$ satisfies the conditions on $T_{1}$ in the following Theorems 2 and 3.

2. Some properties of $\mathscr{M}$ and $\mathscr{B}$. It is readily verified that $\mathscr{T}_{f}$ is a subalgebra of $B[V]$. Moreover, $\mathscr{M}$ is open, [3].

Lemma 1. For $T \in \mathscr{T}_{f}, T \in \mathscr{M}$ if and only if $(f)_{T}=(f)$ and $T$ is invertible.

Proof. If $T$ is invertible and $(f)_{T}=(f)$, it suffices to show that $T^{-1} \in \mathscr{T}_{f}$ in order to conclude that $T \in \mathscr{M}$. Since $T$ is bounded, $T^{-1}$ is bounded. If $\alpha \in(f)$, then let $\eta=T^{-1}(\alpha)$. Then $\alpha=T(\eta)$ implies $\eta \in(f)_{T}=(f)$. Now $T^{-1}(\alpha) \in(f)$ implies $\alpha \in(f)_{T^{-1}}$. Hence, $(f) \subseteq(f)_{T^{-1}}$ or $T^{-1} \in \mathscr{T}_{f}$. Conversely, if $T \in \mathscr{M}$, then $T$ is invertible. Since $T \in \mathscr{T}_{f},(f) \subseteq(f)_{T}$. For $\alpha \in(f)_{T}, \quad T(\alpha)=\eta \in(f)$. Hence, $\alpha=T^{-1}(\eta) \in(f)$, since $T^{-1} \in \mathscr{M}$. Thus $(f)_{T} \subseteq(f)$ and $(f)_{T}=(f)$.

The following lemma provides sufficient conditions for $T \in V$ to be an element of $\mathscr{B}$.

Lemma 2. Let $T \in \mathscr{T}_{f}$ be such that

(i) $S=\operatorname{ker} T \neq\{\theta\}$ is such that $V=S \oplus T(V)$,

(ii) there is a bounded projection $P$ of $V$ onto $S$,

(iii) $(f)_{T}=(f)$.

Then $T \in \mathscr{B}$.

Proof. Define $T_{n}$ on $V$ by $T_{n}(\alpha)=T(\alpha)+P(\alpha) / n$. Then $T_{n} \in \mathscr{T}_{f}$ for each $n$ since the sum of bounded conservative operators is a bounded conservative operator.

Now for $\gamma \in V$, there exists $\alpha \in T(V), \beta \in S$ such that $\gamma=\alpha+\beta$. Since $\alpha \in T(V)$, there is a $\xi \in V$ such that $\alpha=T(\xi)$. But $\xi \in V$ implies $\xi=\delta+\eta$ for some $\delta \in T(V)$ and $\eta \in S$, and $\eta \in S$ implies $T(\eta)=\theta$. Hence, $\gamma=T(\delta)+$ $P(n \beta) / n$ or $\gamma=T_{n}(\delta+n \beta)$ and so $T_{n}$ is onto $V$ for each $n$. Also, $T_{n}(\alpha)=\theta$ implies $T(\alpha)=-P(\alpha) / n$ and thus $T(\alpha) \in S$. Hence, $T(\alpha)=\theta$ and $P(\alpha)=\theta$ implying $\alpha \in \operatorname{ker} P$ and $\alpha \in S$. Therefore, $\alpha=\theta$ and $T_{n}$ is one-to-one for each $n$. Hence $T_{n}^{-1}$ exists for each $n$.

Now $T_{n}(\alpha) \in(f)$ implies $T(\alpha)+P(\alpha) / n \in(f)$. But $P(\alpha) / n \in(f)$ for $\alpha \in V$ and thus $T(\alpha) \in(f)$. Now (iii) implies $\alpha \in(f)$. Since $T_{n}$ is conservative for each $n$, then $(f)_{T_{n}}=(f)$. Thus $T_{n} \in \mathscr{M}$ for each $n$.

Now $\lim _{n \rightarrow \infty} T_{n}^{n}(\alpha)=T(\alpha)$ for all $\alpha \in V$. That is, $\left\{T_{n}\right\}_{n=1}^{\infty}$ converges to $T$ and hence $T \in \bar{M}$. But ker $T \neq\{\theta\}$ implies $T^{-1}$ does not exist and hence $T \notin \mathscr{M}$. Therefore, $T \in \mathscr{B}$. 
Even though the hypotheses of Lemma 2 seem rather strong, (i) is not satisfied even for finite dimensional $V$ whereas (i) and (ii) are both satisfied if $T$ is not the identity transformation and is a bounded projection on $V$. Moreover, (ii) is satisfied for $S=\operatorname{ker} T$ being finite dimensional [1]. It is readily seen that condition (iii) is not a necessary condition for $T$ to be in $\mathscr{B}$, since it is not satisfied by the null operator $Z$ and $Z \in \mathscr{B}$. A necessary condition for $T$ to be in $\mathscr{B}$ is given in [3, Problem 39, p. 267]. An equivalent condition for (ii) of Lemma 2 is given by Lemma 3.

Lemma 3. Let $V$ denote a normed linear space where $V=S \oplus U$. There exists a bounded projection, $P$, of $V$ onto $U$ along $S$ if and only if there exists a real number $k$ such that $\|\gamma\|,\|\xi\| \leqq k$ when $\gamma \in U, \xi \in S$ and $\|\gamma+\xi\|=1$.

Attention is now turned to results motivated by the results found in [2].

Lemma 4. For $T_{1}, T_{2} \in \bar{M}, T_{1} T_{2} \in \overline{\mathscr{M}}$.

Proof. If $T_{1}, T_{2} \in \bar{M}$, then there exist sequences of elements in $\mathscr{M}$ which converge to $T_{1}$ and $T_{2}$ respectively. Clearly the product sequence converges to $T_{1} T_{2}$, implying $T_{1} T_{2} \in \bar{M}$.

Lemma 5. If $T_{1} \in \mathscr{M}, T_{2} \in \mathscr{B}$, then $T_{1} T_{2} \in \mathscr{B}$.

Proof. By Lemma 4, $T_{1} T_{2}$ is in $\overline{\mathscr{M}}$. Now $T_{1} T_{2} \in \mathscr{M}$ and $T_{1}^{-1} \in \mathscr{M}$ implies $T_{2} \in \mathscr{M}$, contradicting $T_{2} \in \mathscr{B}$, since $\mathscr{M}$ is open. Hence, $T_{1} T_{2} \in \mathscr{B}$.

THEOREM 1. $\mathscr{B}$ is a multiplicative semigroup.

Proof. It suffices to show that $\mathscr{B}$ is closed under multiplication. If $T_{1}, T_{2} \in \mathscr{B}$, then $T_{1}, T_{2} \in \overline{\mathscr{M}}$ and by Lemma $4, T_{1} T_{2} \in \overline{\mathscr{M}}=\mathscr{M} \cup \mathscr{B}$. Suppose $T_{1} T_{2} \in \mathscr{M}$. Then $T_{1} T_{2}$ is invertible. Thus $T_{1}$ is onto and so $T_{1} \notin \mathscr{B}$. Hence the supposition is false and $T_{1} T_{2} \in \mathscr{B}$.

The following results pertain to one-to-one elements of $\mathscr{B}$. These elements cannot be range closed nor onto $V$ [3, p. 267].

THEOREM 2. Let $T_{1} \in \mathscr{B}$ be one-to-one. For $T_{2} \in \mathscr{T}_{f}$, if

(i) $T_{2}(V) \subseteq T_{1}(V)$,

(ii) $T_{1}^{-1} T_{2}$ satisfies (i), (ii) of Lemma 2 ,

(iii) $T_{2}((f))=T_{1}((f))$,

(iv) $(f)_{T_{2}}=(f)$,

then $T_{2} \in \mathscr{B}$.

Proof. Let $T=T_{1}^{-1} T_{2} . T_{1}$ being one-to-one and (i) implies that $T$ is well defined. If $x \in(f)$, then $T_{2}(x) \in(f)$ and $T_{2}(x) \in T_{2}((f))=T_{1}((f))$. Thus it follows that $T_{1}^{-1} T_{2}(x) \in(f)$ or $x \in(f)_{T}$. Hence, $(f) \subseteq(f)_{T}$. 
If $x \in(f)_{T}$, then $T_{1}^{-1} T_{2}(x) \in(f)$. Thus $T_{2}(x) \in(f)_{T_{1}-1} \subset(f)$, since $T_{1}$ is one-to-one. Thus $x \in(f)_{T_{2}}=(f)$ and $(f)_{T} \subseteq(f)$. Now $(f)_{T}=(f)$ and (ii) implies by Lemma 2 that $T \in \mathscr{B}$. Thus by Theorem $1, T_{2}=T_{1} T \in \mathscr{B}$.

In the above proof, $T \in B[V]$ follows from the closed graph theorem since $T_{1}^{-1} T_{2}$ is closed. Also it is readily seen that condition (iv) can be replaced by the weaker condition that $T_{2}(x) \in(f)_{T_{1}{ }^{-1}}$ implies that $x \in(f)$.

If $T_{2}$ is one-to-one and in the Banach algebra $\Delta$ of [2] and $(f)_{T_{2}}=(f)$, then $T_{2} \in \mathscr{M}$. Hence in the following theorem condition (iv) of Theorem 2 will be replaced by the condition that $T_{2}$ is one-to-one, which eliminates the need of condition (ii).

THEOREM 3. Let $T_{1} \in \mathscr{B}$ be one-to-one. For $T_{2} \in \mathscr{T}_{f}$, if

(i) $T_{2}(V)=T_{1}(V)$,

(ii) $T_{2}$ is one-to-one,

(iii) $T_{2}((f))=T_{1}((f))$,

then $T_{2} \in \mathscr{B}$.

Proof. Let $T=T_{1}^{-1} T_{2} . T$ is well defined. If $x \in(f)$, then $T_{2}(x) \in(f)$. Hence $T_{2}(x) \in T_{2}((f))=T_{1}((f))$. Thus $T_{1}^{-1} T_{2}(x) \in(f)$ and so $(f) \subseteq(f)_{T}$.

If $x \in(f)_{T}$, then $T_{1}^{-1} T_{2}(x) \in(f)$ implying $T_{2}(x) \in(f)_{T_{1}-1}=T_{1}((f))$, since $T_{1}$ is one-to-one. Thus, by (iii), $T_{2}(x) \in T_{2}((f))=(f)_{T_{2}-1}$, since $T_{2}$ is oneto-one. Hence $x \in(f)$ and $(f)_{T} \subseteq(f)$. Now $(f)_{T}=(f)$ and $T$ being invertible implies that $T \in \mathscr{M}$. Thus by Lemma $5, T_{2}=T_{1} T \in \mathscr{B}$.

Note by Lemma 5 , for any real number $\alpha$ and $T \in \mathscr{B}, \alpha T=(\alpha I) T \in \mathscr{B}$, where $I$ denotes the identity element in $B[V]$. Thus it might be suspected that $\mathscr{B}$ is a linear space. This is not so by the following theorem.

THEOREM 4. If there exists a nonzero $\alpha \in(f)$, then $\mathscr{B}$ is nonconvex.

Proof. Now there exists a bounded projection $P_{1}$ of $V$ onto $\langle\alpha\rangle$ along $S=\operatorname{ker} P_{1} . P_{1}$ is conservative since $\langle\alpha\rangle \subseteq(f)$. Also, $P_{2}=I-P_{1}$ is a bounded conservative projection. It is clear that $(f)_{P_{2}}=(f)$ since $P_{2}(\alpha) \in(f)$ implies $\alpha-P_{1}(\alpha) \in(f)$. Thus, by Lemma 2 it follows that $P_{2} \in \mathscr{B}$.

Now for any $\varepsilon$ such that $0<\varepsilon<1$ consider $P_{1}+\varepsilon I$. $P_{1}+\varepsilon I$ is bounded since $P_{1}$ and $\varepsilon I$ are, and $P_{1}+\varepsilon I$ is onto. Also, $\left(P_{1}+\varepsilon I\right)(\gamma)=\theta$ if and only if $P_{1}(\gamma)=-\varepsilon \gamma$ implying $P_{1}(\gamma)=P_{1}^{2}(\gamma)=-\varepsilon P_{1}(\gamma)$ or $(1+\varepsilon) P_{1}(\gamma)=\theta$. Thus, $P_{1}(\gamma)=\theta$ and so $\varepsilon I(\gamma)=\theta$ or $\gamma=\theta$. Hence $\left(P_{1}+\varepsilon I\right) \in \mathscr{M}$. Now

implies $P_{1} \in \mathscr{B}$.

$$
\left\|P_{1}-\left(P_{1}+\varepsilon I\right)\right\|=\|\varepsilon I\|=\varepsilon\|I\|=\varepsilon
$$

Now it follows that $\mathscr{B}$ is nonconvex since $\frac{1}{2} P_{1}+\frac{1}{2} P_{2}=\frac{1}{2} I \in \mathscr{M}$.

3. Further partition of $\mathscr{T}_{f}$. Let $\mathscr{N}$ denote the complement of $\overline{\mathscr{M}}$ in $\mathscr{T}_{f}$. The following theorem locates the product $T_{1} T_{2}$ where $T_{1} \in \mathscr{N}$ and $T_{2}$ is in either $\mathscr{M}, \mathscr{B}$ or $\mathscr{T}_{f}$. 
THEOREM 5. Let $T_{1} \in \mathscr{N}$.

(i) If $T_{2} \in \mathscr{M}$, then $T_{2} T_{1}$ and $T_{1} T_{2} \in \mathscr{N}$.

(ii) If $T_{2} \in \mathscr{T}_{f}$ and $T_{1}$ is not $1-1$, then $T_{2} T_{1} \in \overline{\mathscr{N}}$.

(iii) If $T_{2} \in \mathscr{T}_{f}$ and $T_{1}$ is not onto, then $T_{1} T_{2} \in \overline{\mathscr{N}}$.

(iv) If $T_{2} \in \mathscr{T}_{f}$ and $(f)_{T_{1}} \neq(f)$, then $T_{2} T_{1} \in \overline{\mathscr{N}}$.

(v) $T_{2} \in \mathscr{B}$ does not imply that $T_{2} T_{1}$ or $T_{1} T_{2}$ is in $\mathscr{N}$.

Proof. (i) If $T_{2} T_{1} \in \bar{M}$, then $T_{2}^{-1}\left(T_{2} T_{1}\right)=T_{1} \in \bar{M}$ contradicting $T_{1} \in \mathscr{N}$. Hence, $T_{2} T_{1} \in \mathscr{N}$. Similarly $T_{1} T_{2} \in \mathscr{N}$.

(ii) If $T_{1}$ is not $1-1$, then $T_{2} T_{1}$ is not $1-1$ implying that $T_{2} T_{1} \notin \mathscr{M}$. Hence $T_{2} T_{1} \in \overline{\mathscr{N}}$.

(iii) If $T_{1}$ is not onto, then $T_{1} T_{2}$ is not onto implying $T_{1} T_{2} \notin \mathscr{M}$. Hence $T_{1} T_{2} \in \bar{N}$.

(iv) Now $(f)_{T_{1}} \subseteq(f)_{T_{2} T_{1}}$. Thus $(f)_{T_{1}} \neq(f)$ implies $(f)_{T_{2} T_{1}} \neq(f)$ and thus $T_{2} T_{1} \notin \mathscr{M}$. Hence $T_{2} T_{1} \in \bar{N}$.

(v) Since the null operator $Z \in \mathscr{B}$ and $T_{1} Z=Z T_{1}=Z, T_{2} \in \mathscr{B}$ does not imply that $T_{1} T_{2}$ or $T_{2} T_{1}$ is in $\mathscr{N}$.

As in [2] the question arises as to whether or not $\mathscr{N}$ is a multiplicative semigroup. In the setting of this paper the answer is no. This is seen by taking $V$ to be the Banach space of bounded real sequences and taking

$$
T_{1}=\left[\begin{array}{cccccc}
0 & 0 & 0 & . & . & . \\
1 & 0 & 0 & . & . & . \\
0 & 1 & 0 & . & . & . \\
. & . & . & . & . & . \\
. & . & . & . & . & .
\end{array}\right], \quad T_{2}=\left[\begin{array}{cccccc}
0 & 1 & 0 & . & . & . \\
0 & 0 & 1 & . & . & . \\
0 & 0 & 0 & 1 & . & . \\
. & . & . & . & . & . \\
. & . & . & . & . & .
\end{array}\right] .
$$

Then $T_{1}, T_{2} \in \mathscr{N}$ and $T_{2} T_{1}=I \in \mathscr{M}$.

4. Triangular matrix summability methods. In this section attention is turned to questions raised in [2]. Consider the sequence of infinite matrices $\{C(k)\}$, where $c_{i j}(k)=\alpha_{i}$, if $i=j$ and $i \leqq k ; c_{i j}(k)=1 / k$, if $i=j$ and $i>k$; $c_{i j}(k)=0$ otherwise; and $\left\{\alpha_{i}\right\}$ is a null sequence with $\alpha_{i} \neq 0$. Then each $C(k)$ is in the maximal group since each $C(k)$ is a triangle and sums only convergent sequences. It follows that $\lim _{k} C(k)=B=\left[b_{i j}\right]$, where $b_{i j}=\alpha_{i}$, $i=j$, and $b_{i j}=0$ otherwise. Hence $B \in \mathscr{B}$.

Consider the matrix $C=\left[c_{i j}\right]$, where $c_{i j}=c$ for $i=j$ and $c_{i j}=1$ for $i=j+2$ and $c_{i j}=0$ otherwise. It was shown in Theorem 5 of [2] that $C \in \mathscr{M}$ if $c>1, C \in \mathscr{B}$ if $c=1$ and $C \in \mathscr{N}$ if $0 \leqq c<1$. Now for $C \in \mathscr{N}$ and $B \in \mathscr{B}$, as in the preceding paragraph, $B C \in \mathscr{B}$. This can be seen by taking the sequence of matrices $\{D(k)\}$ from $\mathscr{B}$ defined by $D(k)=\left[d_{i j}(k)\right]$, where 
$d_{i j}(k)=c \alpha_{i}$, for $i=j$ and $d_{i j}(k)=\alpha_{i}$ for $i=j+2$ and $i \leqq k$ and $d_{i j}(k)=0$ otherwise. Then $\lim _{k} D(k)=B C$. This answers Conjecture 1 in [2].

Also, if the sequence $\left\{\alpha_{n}\right\}$ in the definition of $B$ is taken to be $\{1 / n\}$ and $C \in \mathscr{N}$ as in the above paragraph then $B C=\left[h_{i j}\right]$ where $h_{i j}=c / j$ for $i=j, h_{i j}=1 / j+2$ for $i=j+2$ and $h_{i j}=0$ otherwise. Thus for $t=\left\{t_{n}\right\}$, where $t_{2 n}=0, t_{2 n-1}=(-1)^{n-1},(2 n-1) c^{n-1}, t(B C)=\{0\}$. This answers Conjecture 4(i) in [2].

\section{REFERENCES}

1. Mahlon M. Day, Normed linear spaces, Ergebnisse der Mathematik und ihrer Grenzgebiete, Heft 21, Springer-Verlag, Berlin, 1958. MR 20 \#1187.

2. B. E. Rhoades, Triangular summability methods and the boundary of the maximal group, Math. Z. 105 (1968), 284-290. MR 37 \#4461.

3. Albert Wilansky, Functional analysis, Blaisdell, New York, 1964. MR 30 \#425.

4. - Topological divisors of zero and Tauberian theorems, Trans. Amer. Math. Soc. 113 (1964), 240-251. MR 29 \#6222.

Department of Mathematics, Louisiana Tech University, Ruston, Louisiana 71270 